\title{
ANALYSIS OF TRANSMISSION LINES LOADED WITH PAIRS OF COUPLED RESONANT ELEMENTS AND APPLICATION TO SENSORS
}

\author{
J. Naqui, L. Su, J. Mata, F. Martín \\ GEMMA/CIMITEC, Departament d'Enginyeria Electrònica, Universitat Autònoma de \\ Barcelona, 08193 Bellaterra (Barcelona), Spain \\ Ferran.Martin@uab.es
}

Keywords: Metamaterials, split ring resonators (SRR), stepped impedance resonators (SIR), microstrip line, coplanar waveguides (CPW), microwave sensors, magnetic coupling.

Corresponding Author: Lijuan Su, Departament d'Enginyeria Electrònica, Universitat Autònoma de Barcelona, 08193 Bellaterra (Barcelona), Spain; phone: 349358135 22; e-mail: lijuan.suri.su@gmail.com.

\begin{abstract}
This paper is focused on the analysis of transmission lines loaded with pairs of magnetically coupled resonators. We have considered two different structures: (i) a microstrip line loaded with pairs of stepped impedance resonators (SIRs), and (ii) a coplanar waveguide (CPW) transmission line loaded with pairs of split ring resonators (SRRs). In both cases, the line exhibits a single resonance frequency (transmission zero) if the resonators are identical (symmetric structure with regard to the line axis), and this resonance is different to the one of the line loaded with a single resonator due to inter-resonator coupling. If the structures are asymmetric, inter-resonator coupling enhances the distance between the two split resonance frequencies that arise. In spite that the considered lines and loading resonators are very different and are described by different lumped element equivalent circuit models, the phenomenology associated to the effects of coupling is exactly the same, and the resonance frequencies are given by identical expressions. The reported lumped element circuit models of both structures are validated by comparing the circuit simulations with extracted parameters with both electromagnetic simulations and experimental data. These structures can be useful for the implementation of microwave sensors based on symmetry properties.
\end{abstract}




\section{Introduction}

Artificial transmission lines inspired by metamaterial concepts, also called metamaterial transmission lines, have been the subject of an intensive research in the last decade. These lines are implemented by loading a host line (e.g., a microstrip line or a coplanar waveguide -CPW) with reactive elements, namely inductors, capacitors, and/or resonant elements. Thanks to the presence of these reactive loading elements, there are more degrees of freedom for design purposes (as compared to ordinary transmission lines), and the implementation of microwave components with reduced size and high performance, or the design of devices exhibiting novel functionalities, is possible. There are many reported examples of microwave components based on metamaterial transmission lines, such as enhanced bandwidth components [1-4], multiband components [5-8], leaky-wave antennas with end-fire to back-fire scanning capability [9-12], compact and multiband filters [13-16], couplers [17-18], etc. In most of these implementations, the key aspect to achieve the required device performance or functionality is the controllability of both the characteristic impedance and the dispersion in the constitutive metamaterial transmission lines. Thus, the design of metamaterial-inspired planar microwave components is mainly based on impedance and dispersion engineering [19].

Many of the previous metamaterial based components have been implemented by means of the so-called resonant-type approach, where a host line is loaded with resonant elements, typically split ring resonators (SRR) [20], complementary split ring resonators (CSRRs) [21], open SRRs and CSRRs [22], etc., and, eventually, other reactive elements (inductors or capacitors). The former resonant-type metamaterial transmission line was implemented by loading a CPW transmission line with split ring resonators [20]. This line exhibits a stop band functionality that may be interpreted as due to the extreme values of the effective permeability of the line in the vicinity of the first SRR resonance (positive below it, and negative above it). However, by adding shunt connected inductive elements (strips), it was found that the stop band switches to a pass band with backward (left-handed) wave propagation at low frequencies and forward wave propagation at high frequencies (i.e., the SRR/strip loaded CPW exhibits a composite right/left handed - CRLH - behavior, similar to transmission lines loaded with series capacitances and shunt inductances [23]).

In spite that the behavior of SRR or SRR/strip loaded CPW transmission lines can be interpreted from the theory of metamaterials, it is useful to analyze these lines, and other metamaterial transmission lines, from their lumped element equivalent circuit model, valid as long as the unit cell of these lines is electrically small. In this regard, the former model of SRR loaded lines was reported in [20], and later revised in [24] to account for the effects of the inductive strips on the transmission zero frequency, present below the first transmission band. In these models, coupling between SRRs of adjacent cells was not included, and the orientation of the SRRs was considered to be with their symmetry plane orthogonal to the line axis. With such orientation, the single coupling mechanism between the line and the SRRs is magnetic. However, by rotating the SRRs, electric coupling must also be accounted for in the model (giving rise to mixed coupling), and it explains the asymmetry in the reflection coefficients measured from the two ports, as demonstrated in [25] (an extensive study on the coupling mechanisms between SRRs and the effects of relative orientation is reported in [26],[27]). Coupling between adjacent SRRs was considered in [28], [29]. In [29] it was demonstrated that stop band bandwidth enhancement in SRR-loaded CPW transmission lines is due to the presence of complex modes [30], which appear as conjugate pairs and do not carry net power. 
In the present paper, we study the effects of coupling between the SRRs forming the pair in a CPW transmission line, neglecting the coupling between SRRs of adjacent cells. Moreover, orthogonal orientation between the symmetry plane of the CPW and the symmetry plane of the pair of SRRs is considered. Although for the implementation of metamaterial transmission lines the pairs of SRRs are implemented with identical resonators, disruption of symmetry may be of interest in certain applications, such as sensors and comparators based on symmetry properties [31]. Therefore, we will consider the more general case of CPWs with asymmetric SRR loading (i.e., with different SRRs forming the pair, including different inductive coupling between the line and the resonators). Moreover, since microstrip lines loaded with pairs of shunt connected stepped impedance resonators (SIRs) have been found to be useful for sensing purposes [32], we will also study in detail these lines, where coupling between resonators is also present. It is important to highlight that, in spite that SRR-loaded CPWs and SIR-loaded microstrip lines are described by distinct lumped element circuits, the phenomenology associated to inter-resonator coupling is roughly the same in both structures, and the resonance frequencies predicted by the respective models are given by identical expressions.

The paper is organized as follows: in section 2, the models of the SRR-loaded and SIR-loaded lines are presented, and the resonance frequencies predicted by these models are obtained for the general (asymmetric) case, and for specific cases (asymmetric structure but considering identical resonance frequencies for the two resonators, and symmetric structure). In section 3, the models are validated by comparing circuit simulations with electromagnetic simulations and experimental responses obtained in fabricated structures. The potentiality of these structures for sensing is discussed in section 4. Finally, the main conclusions are pointed out in section 5.

\section{Circuit model and analysis}

In this section, the circuit models of the considered structures are reported, and the resonance frequencies, given by the transmission zero frequencies, are inferred. We will first study the CPW loaded with a pair of magnetically coupled SRRs, and then the microstrip line loaded with a pair of coupled (also magnetically) SIRs.

\subsection{CPW loaded with a pair of coupled SRRs}

Figure 1(a) depicts the typical topology (and relevant dimensions) of a CPW loaded with a pair of SRRs, etched in the back substrate side, underneath the slots. The symmetry plane of the SRRs is orthogonal to the line axis, so that electric coupling between the line and the SRRs can be neglected [25], the main coupling mechanism thus being magnetic. Moreover, the SRRs are oriented with the gap of the outer ring located on the opposite side from the interaction region between SRRs (i.e., the vicinity of the line axis). By this means, we ensure that the dominant coupling mechanism between SRRs is magnetic. Moreover, we have considered rectangular shaped SRRs to enhance such inter-resonator coupling. Although the structure depicted in Fig. 1 is symmetric, the lumped element equivalent circuit model, depicted in Fig. 1(b), corresponds to the general case of an asymmetric structure with different SRRs and line-to-resonators couplings (the model is identical to the one reported in [20], but considering asymmetry and inter-resonator coupling). In the model, $L$ and $C$ are the line inductance and capacitance, respectively; the SRRs are described by the resonant tanks $L_{1}-C_{1}$ and $L_{2}-C_{2}$, and their coupling to the line is accounted for by the mutual inductances $M_{1}$ and $M_{2}$; finally, $M^{\prime}$ is the mutual inductance between SRRs. This model is valid in the frequency region of interest (i.e., the vicinity of the fundamental resonance of the SRR) as long as the SRRs are electrically small 
resonant particles (a necessary condition for this is that the two metallic loops forming the SRR are tightly coupled, and hence very close [33, 34]).

(a)

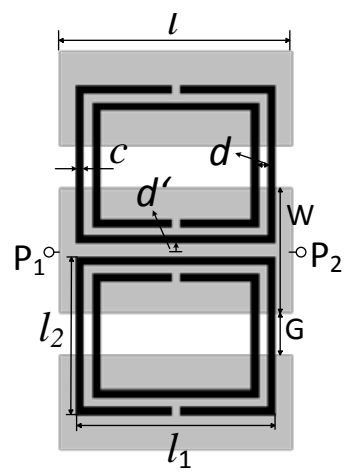

(b)

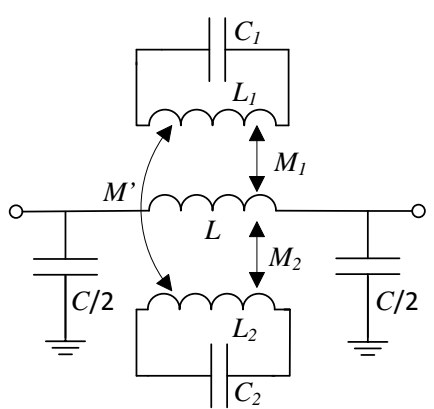

Fig. 1. Typical topology of a CPW loaded with a pair of SRRs (a), and lumped element equivalent circuit model, considering magnetic coupling between SRRs (b). The SRRs (in black) are etched in the back substrate side of the CPW (depicted in grey). The model considers the general case of different SRRs.

The circuit of Fig. 1(b) is a $\pi$-circuit where the shunt branches are purely capacitive. Thus, the transmission zeros (or resonance frequencies of the coupled resonators) are given by the poles of the series reactance. From Kirchhoff's equations, including the different mutual couplings, applied to the series branch of the circuit of Fig. 1(b), the impedance of this branch is found to be:

$$
Z_{s}(\omega)=j \omega L+j \omega^{3} \times\left\{\frac{C_{1} M_{1}^{2}\left(1-\frac{\omega^{2}}{\omega_{2}^{2}}\right)+C_{2} M_{2}^{2}\left(1-\frac{\omega^{2}}{\omega_{1}^{2}}\right)+2 \omega^{2} M_{1} M_{2} M^{\prime} C_{1} C_{2}}{\left(1-\frac{\omega^{2}}{\omega_{1}^{2}}\right)\left(1-\frac{\omega^{2}}{\omega_{2}^{2}}\right)-\omega^{4} M^{\prime 2} C_{1} C_{2}}\right\}
$$

where $\omega_{1}=\left(L_{1} C_{1}\right)^{-1 / 2}$ and $\omega_{2}=\left(L_{2} C_{2}\right)^{-1 / 2}$ are the resonance frequencies of the isolated resonators. By forcing the denominator of the last term to be zero, the notch (or transmission zero) frequencies can be derived, namely:

$$
\omega_{ \pm}^{2}=\frac{\omega_{1}^{2}+\omega_{2}^{2} \pm \sqrt{\left(\omega_{1}^{2}-\omega_{2}^{2}\right)^{2}+4 M^{\prime 2} \omega_{1}^{4} \omega_{2}^{4} C_{1} C_{2}}}{2\left[1-M^{\prime 2} \omega_{1}^{2} \omega_{2}^{2} C_{1} C_{2}\right]}
$$

Note that the mutual coupling $M^{\prime}$ between resonators enhances the distance between transmission zeros, i.e.,

$$
\omega_{+}^{2}-\omega_{-}^{2}=\frac{\sqrt{\left(\omega_{1}^{2}-\omega_{2}^{2}\right)^{2}+4 M^{\prime 2} \omega_{1}^{4} \omega_{2}^{4} C_{1} C_{2}}}{\left[1-M^{\prime 2} \omega_{1}^{2} \omega_{2}^{2} C_{1} C_{2}\right]}>\omega_{1}^{2}-\omega_{2}^{2}
$$

However, the resonance frequencies, $\omega_{+}$and $\omega_{\rightarrow}$, do not depend on the mutual coupling between the line and the SRRs $\left(M_{1}\right.$ and $\left.M_{2}\right)$.

Let us now consider two particular cases of interest. The first one is the symmetric SRR loaded CPW, namely $M_{1}=M_{2}=M, L_{1}=L_{2}=L_{r}$, and $C_{1}=C_{2}=C_{r}$ (and hence $\omega_{1}=\omega_{2}=\omega_{0}$ ). Under these conditions, the two solutions of (2) are

$$
\omega_{ \pm}=\frac{\omega_{o}}{\sqrt{1 \mp \frac{M^{\prime}}{L_{r}}}}=\frac{\omega_{o}}{\sqrt{1 \mp k_{M}}}
$$


where $k_{M}$ is the magnetic coupling coefficient [36]. However, one of the solutions, the one with the $(-)$ sign in the radicand of $(4)\left(\omega_{+}\right)$, is not actually a transmission zero frequency. The reason is that this frequency also nulls the numerator of the last term in (1). By applying the l'Hôpital's rule, it follows that the series impedance is finite at the frequency $\omega_{+}$; hence, we can conclude that only one transmission zero, to the left of $\omega_{0}$ (since $M^{\prime}>0$ ), appears in the transmission coefficient for symmetric structures with magnetically coupled SRRs.

The second case of interest is the asymmetric structure (different coupled SRRs), but considering that their fundamental resonance frequencies are identical, that is, $L_{1} \neq L_{2}, C_{1} \neq C_{2}$, $\omega_{1}=\omega_{2}=\omega_{0}$. In this case, expression (2) gives:

$$
\omega_{ \pm}=\frac{\omega_{o}}{\sqrt{1 \mp \frac{M^{\prime}}{\sqrt{L_{1} L_{2}}}}}=\frac{\omega_{o}}{\sqrt{1 \mp k_{M}}}
$$

Note that (5) is formally identical to (4). The two frequencies given by (5) null the denominator of the last term in (1), but, in general, none of them null the numerator. However, if the following condition is satisfied

$$
C_{1} M_{1}^{2}+C_{2} M_{2}^{2}=2 M_{1} M_{2} \sqrt{C_{1} C_{2}}
$$

then the numerator of the last term in (1) is also null at $\omega_{+}$, and the impedance of the series branch is finite at this frequency (as the application of the l'Hôpital's rule reveals). Expression (6) can be simplified to:

$$
\frac{M_{1}}{M_{2}}=\sqrt{\frac{L_{1}}{L_{2}}}
$$

Thus, if condition (7) is satisfied, then only one transmission zero (at $\omega_{-}$) is expected (note that (7) represents a balance that forces the structure to behave similarly to the symmetric one). Otherwise, two transmission zeros at the frequencies given by (5), i.e., one above and the other below $\omega_{0}$, are expected.

\subsection{Microstrip line loaded with a pair of coupled SIRs}

The typical topology, and lumped element equivalent circuit model, of a microstrip line loaded with a pair of SIRs (a resonator used in [35] for the implementation of band pass filters) are depicted in Fig. 2. Even for wide (low impedance) host microstrip lines, the coupling between SIRs cannot be neglected and it has been found that such coupling is magnetic and negative [32], as indicated in Fig. 2(b). The negative sign is consequence of the currents flowing in opposite directions in both resonant elements for the symmetric case. We have opted to designate such coupling as $-\left|M^{\prime}\right|$ to avoid any confusion related to the sign. The circuit parameters $L$ and $C$ are the inductance and capacitance of the line, respectively, and the SIRs, in general of different dimensions, are described by the series resonators $L_{1}-C_{1}$ and $L_{2}-C_{2}$. Using the well-known transformation indicated in Fig. 3(a) [36], the lumped element equivalent circuit model can be depicted as shown in Fig. 3(b), where the mutual coupling between inductive elements is suppressed. The circuit of Fig. 3(b) is a T-model where the series branches are purely inductive. Thus, the transmission zeros (or resonance frequencies of the coupled resonators) are given by the zeros of the shunt reactance. Excluding the capacitance $C$ (which does not affect the zeros of the shunt reactance), the impedance of the shunt branch is given by: 


$$
Z_{p}(\omega)=\frac{C_{1} C_{2} \omega^{4}\left\{\left|M^{\prime}\right|^{2}-L_{1} L_{2}\right\}+\omega^{2}\left\{L_{1} C_{1}+L_{2} C_{2}\right\}-1}{j \omega\left\{C_{1} C_{2} \omega^{2}\left(L_{1}+L_{2}+2\left|M^{\prime}\right|\right)-\left(C_{1}+C_{2}\right)\right\}}
$$

Forcing the numerator to be zero, the transmission zero frequencies are given by

$$
\omega_{ \pm}^{2}=\frac{\omega_{1}^{2}+\omega_{2}^{2} \pm \sqrt{\left(\omega_{1}^{2}-\omega_{2}^{2}\right)^{2}+4\left|M^{\prime}\right|^{2} \omega_{1}^{4} \omega_{2}^{4} C_{1} C_{2}}}{2\left[1-\left|M^{\prime}\right|^{2} \omega_{1}^{2} \omega_{2}^{2} C_{1} C_{2}\right]}
$$

where $\omega_{1}=\left(L_{1} C_{1}\right)^{-1 / 2}$ and $\omega_{2}=\left(L_{2} C_{2}\right)^{-1 / 2}$. Note that $M^{, 2}=\left|M^{\prime}\right|^{2}$, and hence expressions (9) and (2), providing the transmission zero frequencies in significantly different structures, are identical.

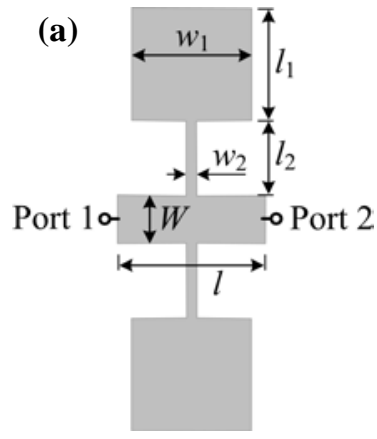

(b)

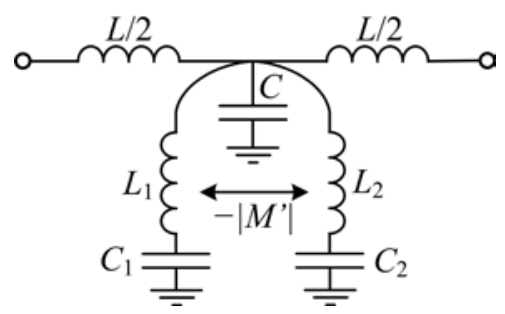

Fig. 2. Typical topology of a microstrip line loaded with a pair of SIRs (a), and lumped element equivalent circuit model, considering magnetic coupling between SIRs (b). The model considers the general case of different SIRs.

(a)
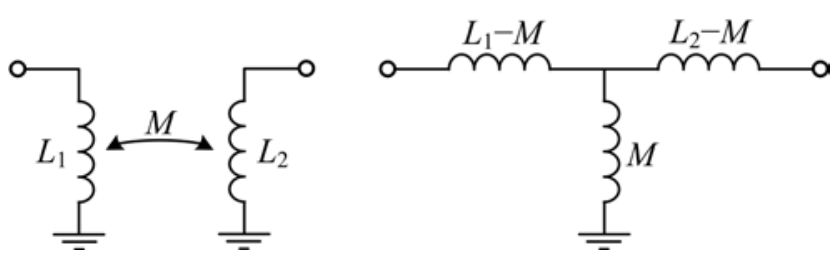

(b)

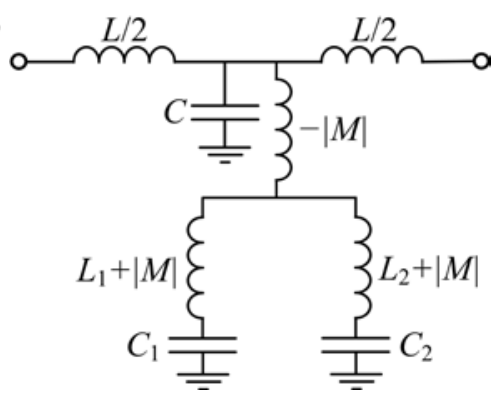

Fig. 3. Two-port with magnetically coupled inductors and its equivalent T-circuit model (a), and model of the SIR loaded line that results by applying the previous equivalence (b).

Obviously, for the symmetric structure $\left(L_{1}=L_{2}=L_{r}, C_{1}=C_{2}=C_{r}\right.$ and $\left.\omega_{1}=\omega_{2}=\omega_{0}\right)$, expression (9) simplifies to (4). However, there is an important difference: in this case (SIR-loaded line), the solution $\omega_{\text {- }}$ nulls the denominator of (8), which means that the valid solution (i.e., the one with physical meaning) is $\omega_{+}$. In other words, for the symmetric case, like in SRR loaded lines, SIR loaded lines exhibit a single transmission zero. However, unlike in SRRs, it appears above the resonance frequency, $\omega_{0}$, of the isolated SIR.

For the asymmetric structure (different coupled SIRs), but considering that their fundamental resonance frequencies are identical $\left(L_{1} \neq L_{2}, C_{1} \neq C_{2}, \omega_{1}=\omega_{2}=\omega_{0}\right)$, the solutions are given by expression (5). However, unlike in SRR loaded lines, in SIR loaded lines there is not a balance condition (similar to the one of expression 7) able to null the denominator of (8) for one of the solutions of (5). In other words, there are always two transmission zeros in SIR loaded lines satisfying $L_{1} \neq L_{2}, C_{1} \neq C_{2}, \omega_{1}=\omega_{2}=\omega_{0}$. Indeed the analysis carried out by introducing (5) in 
the denominator of (8) reveals that this denominator nulls for $\omega_{\text {_ }}$ if the following condition is satisfied:

$$
\frac{C_{1}+C_{2}}{2}=\sqrt{C_{1} C_{2}}
$$

which corresponds to the pure symmetric case. This is an important difference between SRR and SIR loaded lines with coupled resonators. Notice that the balance condition in (7) depends on the mutual coupling $M_{1}$ and $M_{2}$ between the line and the resonators. $M_{1}$ and $M_{2}$ add extra parameters in the model of Fig. 1(b), as compared to the model of Fig. 2(b) or 3(b), and for this reason a balance condition able to give only a single resonance for the considered case $\left(L_{1} \neq L_{2}\right.$, $\left.C_{1} \neq C_{2}, \omega_{1}=\omega_{2}=\omega_{0}\right)$ in SIR loaded lines is not possible.

\section{Validation}

The validation of the models of the previous structures is carried out by extracting circuit parameters and comparing the circuit simulations to electromagnetic simulations and, in some cases, to measurements on fabricated structures.

\section{1. $\quad$ SRR loaded CPWs}

Let us first consider the symmetric case, where SRR and CPW dimensions are set to $l_{1}=4.8 \mathrm{~mm}$, $l_{2}=3.8 \mathrm{~mm}, c=d=0.2 \mathrm{~mm}, l=5.6 \mathrm{~mm}, W=3 \mathrm{~mm}$, and $G=1.01 \mathrm{~mm}$. The lossless electromagnetic simulations (inferred from Agilent Momentum) of the structure, considering different values of inter-resonator distance, $d$ ', are depicted in Fig. 4 (the considered substrate is the Rogers RO3010 with dielectric constant $\varepsilon_{r}=11.2$ and thickness $h=1.27 \mathrm{~mm}$ ). The circuit parameters for the four considered cases (extracted from the method reported in [37]) are shown in Table I. As $d$ ' increases, the mutual coupling $M^{\prime}$ decreases, and the resonance frequency increases, in agreement with the $\omega_{-}$solution of (4). Note that the other circuit parameters do not significantly vary, and the agreement between circuit and electromagnetic simulation in the region of interest is very good, pointing out the validity of the model. We have fabricated these structures, and we have obtained the measured response, also included in Fig. 4 (the details of the SRRs in one of the samples are shown in the inset of Fig. 4a). The agreement is reasonable, taking into account the typical tolerances in the fabrication process (a milling machine has been used) and in the dielectric constant as well. Note also that the electromagnetic and circuit simulations are lossless. Although by excluding losses in electromagnetic and circuit simulation, we obtain less accuracy as compared to measurement, parameter extraction is preferred by excluding losses. Otherwise, too many elements appear in the model. However, the resistive elements modeling losses at the circuit level (i.e., parasitics), which may be included in the resonant elements and eventually in the line model, can be introduced and determined by curve fitting once the reactive parameters are known. Notice, however, that in asymmetric structures this involves two resistances in the resonant elements plus the resistance/s modeling losses in the lines. For this reason, we do not have considered such curve fitting in this work. 
TABLE I. EXTRACTED CIRCUIT PARAMETERS (SYMMETRIC CASE) FOR DIFFERENT VALUES OF $d$ '

\begin{tabular}{|c|c|c|c|c|c|c|}
\hline$d^{\prime}(\mathrm{mm})$ & $L(\mathrm{nH})$ & $C(\mathrm{pF})$ & $C_{r}(\mathrm{pF})$ & $L_{r}(\mathrm{nH})$ & $M(\mathrm{nH})$ & $M^{\prime}(\mathrm{nH})$ \\
\hline 0.105 & 1.82 & 1.58 & 0.44 & 6.85 & 0.82 & 1.74 \\
\hline 0.305 & 1.86 & 1.58 & 0.44 & 6.85 & 0.82 & 1.29 \\
\hline 0.505 & 1.84 & 1.57 & 0.43 & 6.85 & 0.81 & 1.02 \\
\hline 0.755 & 1.85 & 1.55 & 0.43 & 6.85 & 0.80 & 0.80 \\
\hline
\end{tabular}

(a)

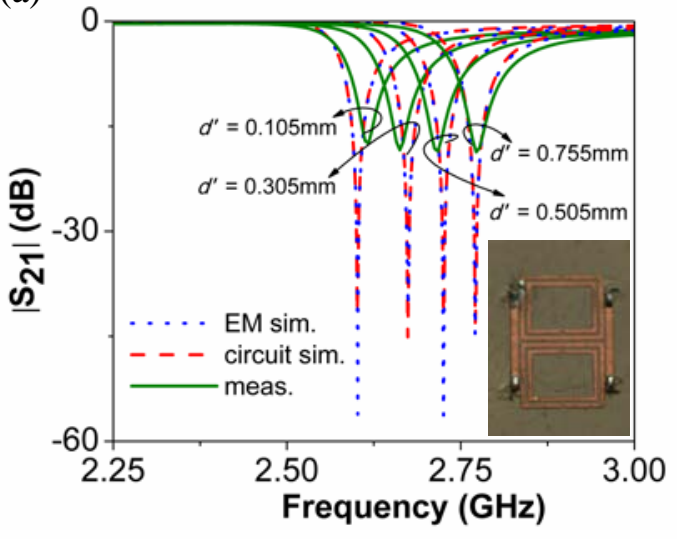

(b)

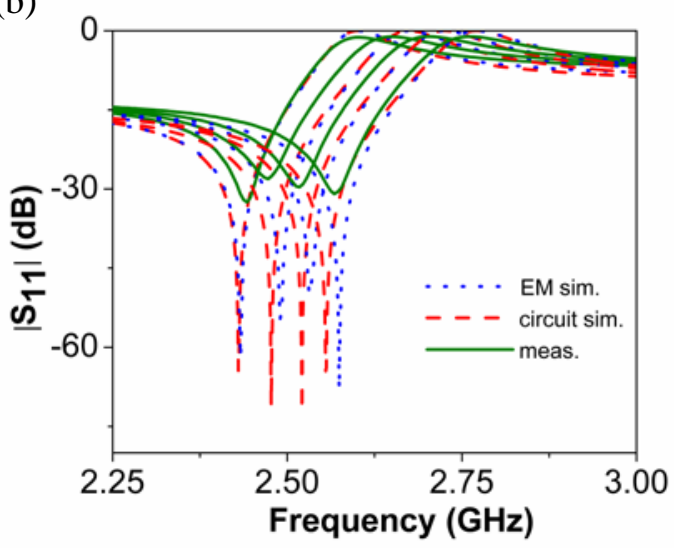

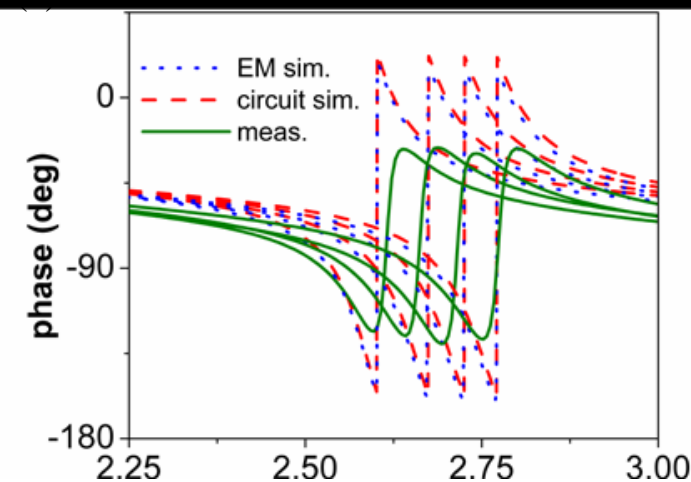

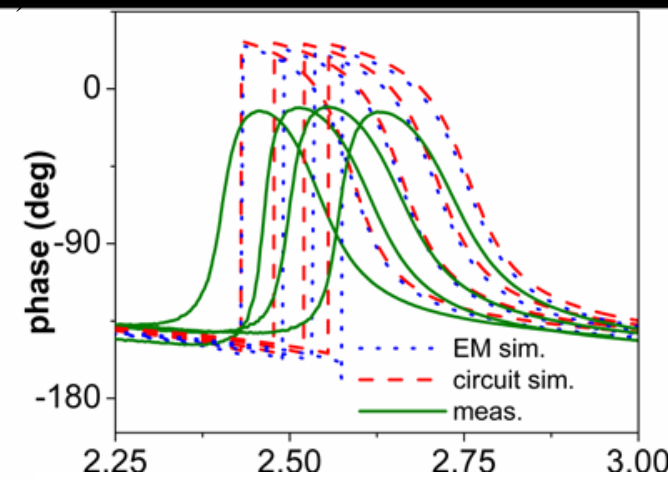

Fig. 4. Frequency response (symmetric case) for different values of $d$ '. (a) Magnitude transmission coefficient, (b) magnitude reflection coefficient, (c) phase transmission coefficient, (d) phase reflection coefficient. The inset of (a) shows a picture of the SRRs (for one of the samples), etched in the back side of the CPW.

Let us now consider the asymmetric SRR-loaded line with identical resonance frequency for both SRRs $\left(\omega_{1}=\omega_{2}=\omega_{0}\right)$. The considered geometrical parameters are: $l=5.6 \mathrm{~mm}, W=3 \mathrm{~mm}, G$ $=1.01 \mathrm{~mm}$; upper SRR: $l_{1}=4 \mathrm{~mm}, l_{2}=3 \mathrm{~mm}, c=0.2 \mathrm{~mm}, d=0.1 \mathrm{~mm}, d^{\prime}=0.305 \mathrm{~mm}$; lower SRR: $l_{1}=4.5 \mathrm{~mm}, l_{2}=4.1 \mathrm{~mm}, c=0.2 \mathrm{~mm}, d=0.725 \mathrm{~mm}, d^{\prime}=0.155 \mathrm{~mm}$. The extracted parameters are: $L=1.77 \mathrm{nH}, C=1.60 \mathrm{pF}, L_{1}=5.0 \mathrm{nH}, C_{1}=0.42 \mathrm{pF}, L_{2}=7.20 \mathrm{nH}, C_{2}=0.29 \mathrm{pF}, M_{1}=0.51 \mathrm{nH}, M_{2}=$ $0.77 \mathrm{nH}, M^{\prime}=0.96 \mathrm{nH}$. As expected, two notches (one above and the other below $\omega_{0}$ ) are present. The agreement between the circuit and electromagnetic simulation is very good, according to Fig. 5. This figure also includes the simulated and circuit response of the CPW loaded with a single resonator, to verify that the resonance frequency is identical in both resonant elements. Moreover, we have verified through circuit simulation that when condition (7) is satisfied, only one notch appears (see Fig. 5). However the verification of this through 
electromagnetic simulation and experiment is difficult since it is not so simple to find out the layout whose extracted parameters exactly satisfy (7).

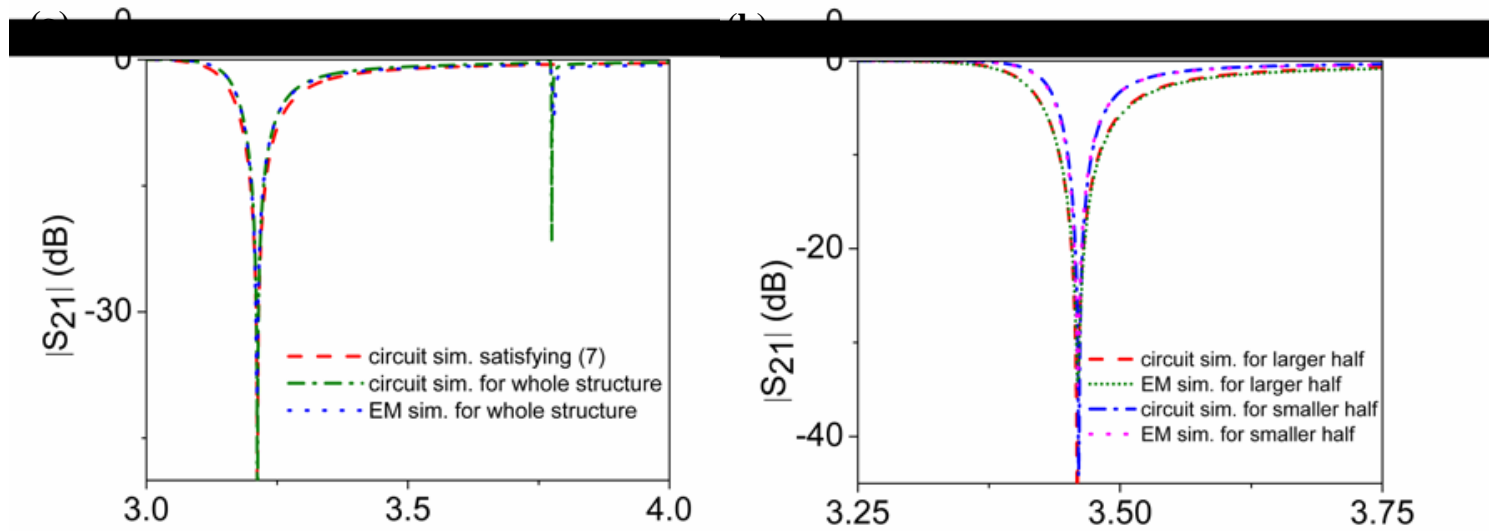

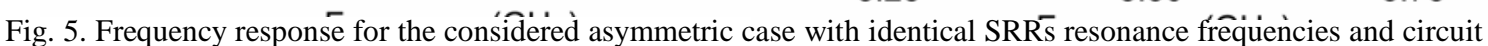
simulation corresponding to $L_{1}=5 \mathrm{nH}, L_{2}=7.2 \mathrm{nH}, M_{2}=0.774 \mathrm{nH} M_{1}=0.645 \mathrm{nH}$, i.e., satisfying (7) (a). The responses of the CPW loaded with only the larger or smaller SRR are depicted in (b).

Finally, we have considered an asymmetric structure with different SRR resonance frequencies. The geometry is as follows: $l=5.6 \mathrm{~mm}, W=3 \mathrm{~mm}, G=1.01 \mathrm{~mm}$; upper SRR: $l_{1}=4.8 \mathrm{~mm}, l_{2}=$ 4.6mm, $c=d=0.2 \mathrm{~mm}$; lower SRRs: $l_{1}=4.8 \mathrm{~mm}, l_{2}=3.8 \mathrm{~mm}, c=d=0.2 \mathrm{~mm}$. We have obtained the frequency response and the circuit parameters for four different values of $d$ ' (see table II). The comparison between the circuit and electromagnetic simulations is depicted in Fig. 6, where good agreement is again obtained, further validating the proposed model. The measured responses (also included in the figure for comparison purposes) reveal that the agreement with circuit and electromagnetic simulations is reasonable. The pair of notches (at $f_{-}=\omega / 2 \pi$ and $f_{+}=$ $\left.\omega_{+} / 2 \pi\right)$ are depicted in Fig. 7, and verify that their distance increases as $d$ ' decreases, in agreement to (3).

TABLE II. EXTRACTED CIRCUIT PARAMETERS (ASYMMETRIC CASE) FOR DIFFERENT VALUES OF $d$,

\begin{tabular}{|c|c|c|c|c|c|c|c|c|c|}
\hline$d^{\prime}(\mathbf{m m})$ & $L(\mathbf{n H})$ & $C(\mathbf{p F})$ & $C_{1}(\mathbf{p F})$ & $L_{1}(\mathbf{n H})$ & $M_{1}(\mathbf{n H})$ & $C_{2}(\mathbf{p F})$ & $L_{2}(\mathbf{n H})$ & $M_{2}(\mathbf{n H})$ & $M^{\prime}(\mathbf{n H})$ \\
\hline 0.105 & 1.87 & 1.58 & 0.37 & 9.85 & 1.00 & 0.44 & 6.85 & 0.83 & 1.91 \\
\hline 0.305 & 1.84 & 1.58 & 0.37 & 9.85 & 0.98 & 0.44 & 6.85 & 0.82 & 1.43 \\
\hline 0.505 & 1.83 & 1.57 & 0.37 & 9.85 & 0.97 & 0.43 & 6.85 & 0.81 & 1.14 \\
\hline 0.755 & 1.86 & 1.55 & 0.37 & 9.85 & 0.93 & 0.43 & 6.85 & 0.80 & 0.90 \\
\hline
\end{tabular}

(a)

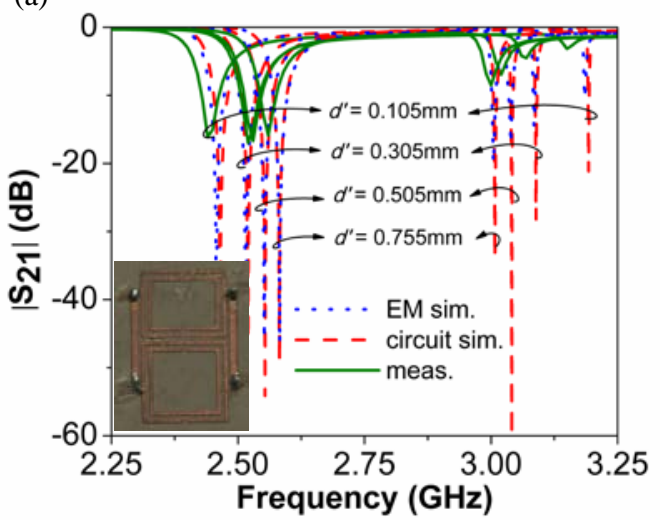

(b)

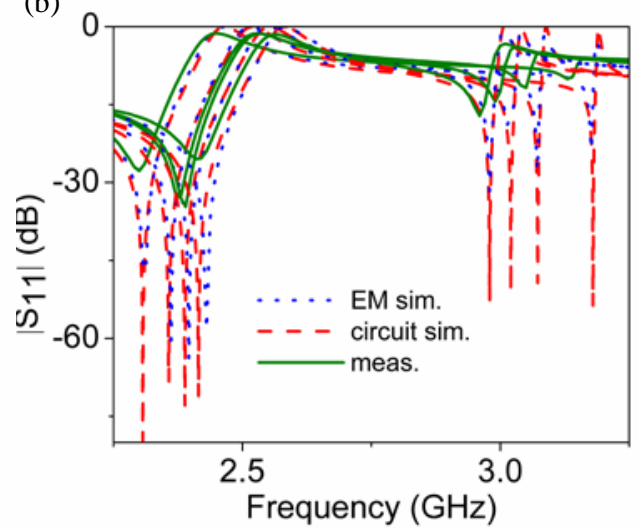


(c)

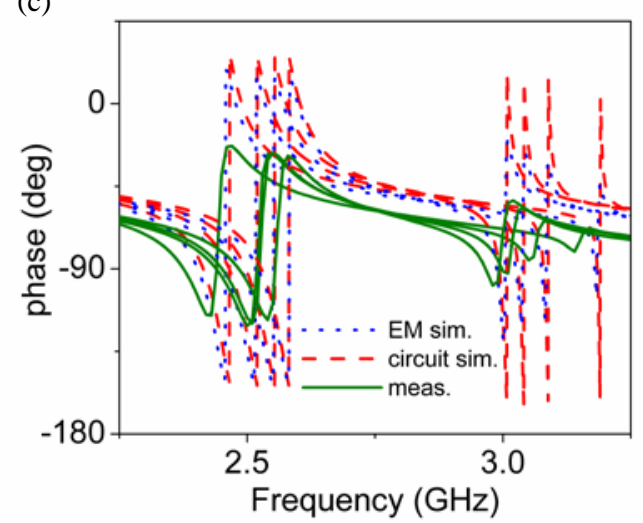

(d)

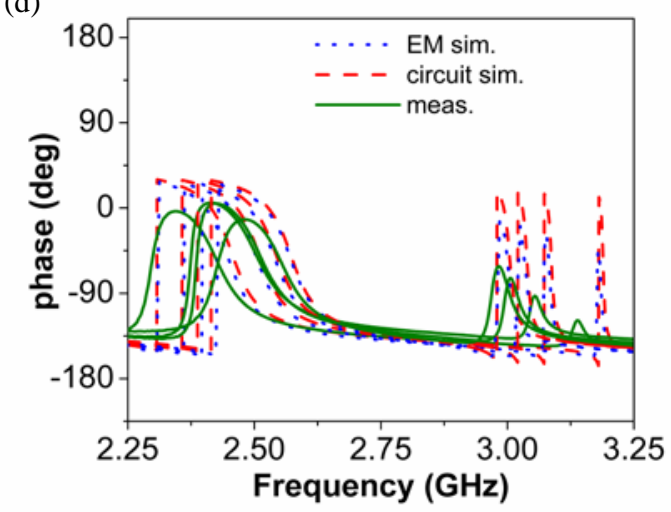

Fig. 6. Frequency response (asymmetric case) for different values of $d^{\prime}$ '. (a) Magnitude transmission coefficient, (b) magnitude reflection coefficient, (c) phase transmission coefficient, (d) phase reflection coefficient. The inset of (a) shows a picture of the SRRs (for one of the samples), etched in the back side of the CPW. To avoid the slot mode, the ground plane regions are connected through vias and metallic strips, also etched in the back side of the substrate (at both sides of the SRRs).

\subsection{SIR loaded microstrip lines}

In this case, we have validated the proposed model by considering three different structures: one symmetric and two asymmetric. These structures have been fabricated on the Rogers RO4003C substrate with dielectric constant $\varepsilon_{r}=3.1$ and thickness $h=812.8 \mu \mathrm{m}$, and are depicted in Fig. 8. The difference between these SIR loaded lines is simply the size of the capacitive patch of the upper SIR. The dimensions for the symmetric structure are: $W=1.83 \mathrm{~mm}, l=15.9 \mathrm{~mm}$, $l_{1}=l_{2}=2.6 \mathrm{~mm}, w_{1}=5.5 \mathrm{~mm}, w_{2}=250 \mu \mathrm{m}$. For the asymmetric structures, we have simply varied $l_{1}$ with $\Delta l_{1}= \pm 0.5 \mathrm{~mm}$. Fig. 9(a) depicts the frequency responses (circuit and electromagnetic simulations) of the microstrip line loaded with only the upper SIR, whereas Fig. 9(b) shows the responses (in this case including also measurement) of the structures of Fig. 8 (i.e., with the coupled SIRs). For the three considered cases the following circuit values have been extracted: $L=1.80 \mathrm{nH}, C=0.57 \mathrm{pF}, L_{1}=L_{2}=2.46 \mathrm{nH}, C_{1}=0.65 \mathrm{pF}$, and $|M|=0.31 \mathrm{nH}$. The capacitance of the upper patch is $C_{2}=0.65 \mathrm{pF}$ (Fig. 8a), $C_{2}=0.80 \mathrm{pF}$ (Fig. 8b), and $C_{2}=0.50 \mathrm{pF}$ (Fig. 8c). The agreement is good in all cases pointing out the validity of the proposed model. For the symmetric structure (Fig. 8a), only one resonance appears, and it is located to the right of the one of the isolated resonator, as expected according to the results of the previous section. For the asymmetric structures, inter-resonator coupling enhances the distance between resonances, as compared to the distance between the resonances of the individual SIRs, also in accordance to the results of the previous section.

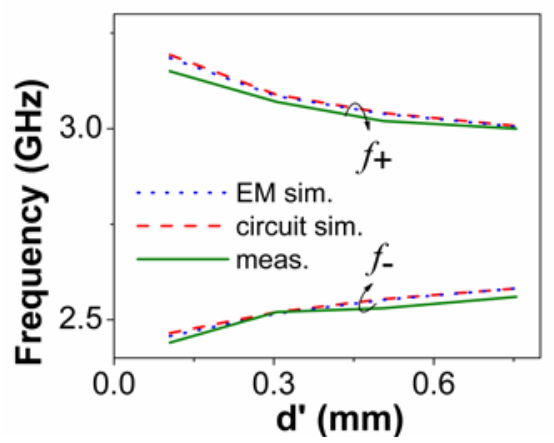

Fig. 7. Variation of the notch frequencies as a function of $d^{\prime}$ for the asymmetric SRR-loaded CPW. 

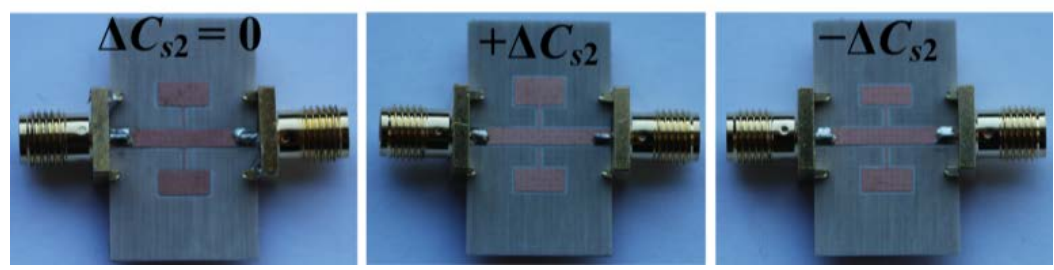

Fig. 8. Photograph of the fabricated SIR loaded lines. (a) Symmetric, (b) asymmetric with larger capacitance of the upper SIR, and (c) asymmetric with smaller capacitance of the upper SIR.

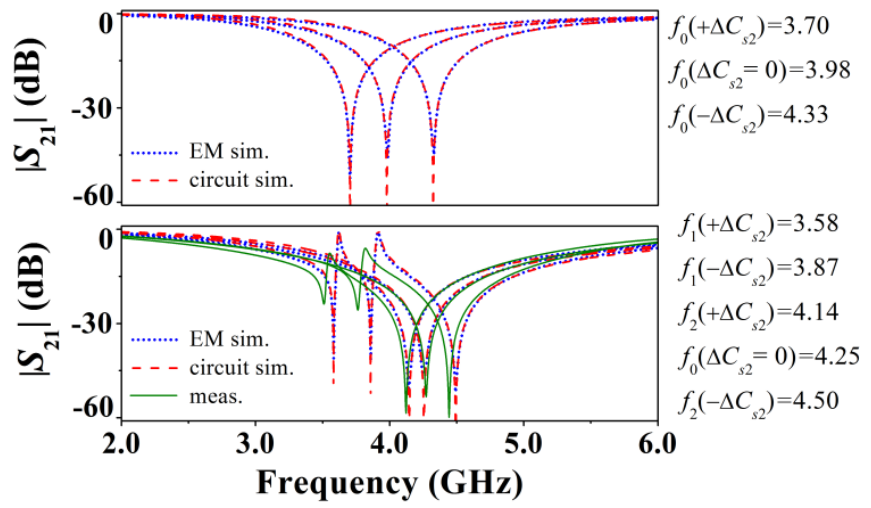

Fig. 9. Transmission coefficient corresponding to the lossless electromagnetic and circuit simulations for a microstrip line loaded (a) with a single SIR (not fabricated), and (b) with a pair of SIRs (fabricated and shown in Fig. 8). In (b), measurements are included. The frequencies indicated in the right correspond to theoretical values.

\section{Potential application to microwave sensors}

The previous structures can be useful for sensing purposes, especially for sensors and comparators based on dielectric loading. The idea is to disrupt the symmetry of a SIR or a SRR loaded transmission line by means of a dielectric load, located on top or in the vicinity of one of the resonant elements. This causes the appearance of two notches in the transmission coefficient and this can be useful for sensing. Note that these structures are of special interest, for instance, for the determination of defects or abnormalities in a substance by comparison to a reference. Indeed, the asymmetric SIR loaded transmission lines of Fig. 8 exhibit different patch capacitance for the upper SIR in order to emulate different dielectric loads between the two resonators forming the pair. According to Fig. 9, this asymmetry produces two notches in the transmission coefficient, which can be easily detected. The experimental validation of this sensing principle was reported in [32], where a conductor backed CPW transmission line loaded with a pair of symmetric SIRs was tested with and without the presence of a piece of glass on the top of one of the SIRs. As expected, a frequency response with two notches in the transmission coefficient, similar to those depicted in Fig. 9 for the asymmetric cases, was obtained. Work is in progress for the implementation of sensors and comparators based on SRR loaded lines.

\section{Conclusions}

In summary, we have studied the transmission properties of transmission lines loaded with pairs of magnetically coupled resonant elements. Specifically, microstrip lines loaded with coupled SIRs and CPWs loaded with coupled SRRs have been considered. In both cases, the lumped element equivalent circuit model of the structure has been proposed. From these models, the transmission zero frequencies (or resonance frequencies of the coupled resonators) have been 
inferred, and it has been found that the general analytical expressions providing such resonances are identical in both structures. The main difference concerns the symmetric case. If the resonators are identical, then only one resonance appears, and this resonance is below and above $\omega_{0}$, the resonance of the isolated resonator, for the SRR and SIR loaded lines, respectively. The second difference concerns the case of distinct resonators but exhibiting the same resonance frequency. In both cases (SIR and SRR loaded lines), two resonance frequencies (one below and the other above $\omega_{0}$ ) appear. However, for SRR loaded lines there is a kind of balance condition that nulls one of these frequencies, whereas such condition does not exist in SIR loaded lines, and the two resonances are always present. The models have been validated by comparing circuit simulations with extracted parameters with electromagnetic simulations and experimental responses obtained in fabricated prototypes. The potentiality of these structures for microwave sensors has been also pointed out.

\section{Acknowledgements}

This work has been supported by MINECO (Spain) under projects TEC2010-17512, CSD200800066, TEC2013-40600-R and AGAUR-Generalitat de Catalunya under project 2009SGR-421. Jordi Naqui is in debt to MECD (Spain) for supporting his work through the FPU grant AP2010-0431. Ferran Martín is in debt to ICREA for supporting his work.

\section{References}

[1] H. Okabe, C. Caloz, T. Itoh, "A compact enhanced bandwidth hybrid ring using an artificial lumped element left handed transmission line section”, IEEE Trans. Microw. Theory Techn., vol. 52, pp. 798804, Mar. 2004.

[2] M.A. Antoniades and G.V. Eleftheriades, "A broadband series power divider using zero-degree metamaterial phase shifting lines”, IEEE Microw. Wirel. Comp. Lett., vol. 15, pp. 808-810, Nov. 2005.

[3] M.A. Antoniades, and G.V. Eleftheriades, "A broadband Wilkinson balun using microstrip metamaterial lines”, IEEE Ant. Wirel. Propag. Lett., vol. 4, pp. 209-212, 2005.

[4] G. Sisó, J. Bonache, M. Gil and F. Martín, "Application of resonant-type metamaterial transmission lines to the design of enhanced bandwidth components with compact dimensions", Microw. Opt. Techn. Lett., vol. 50, pp. 127-134, Jan. 2008.

[5] I-H. Lin, M. DeVincentis, C. Caloz, and T. Itoh, "Arbitrary dual-band components using composite right/left-handed transmission lines”, IEEE Trans. Microw. Theory Techn., vol. 52, pp. 1142-1149, Apr. 2004.

[6] A. C. Papanastasiou, G. E. Georghiou, G. V. Eleftheriades, "A quad-band wilkinson power divider using generalized NRI transmission lines”, IEEE Microw. Wirel. Comp. Lett., vol. 18, pp. 521-523, Aug. 2008.

[7] M. Durán-Sindreu, G. Sisó, J. Bonache, F. Martín, "Planar multi-band microwave components based on the generalized composite right/left handed transmission line concept", IEEE Trans. Microw. Theory Techn., vol. 58, no 12, pp. 3882-3891, Dec. 2010.

[8] M. Durán-Sindreu, J. Bonache, F. Martín and T. Itoh, "Single-layer fully-planar extended-composite right/left handed transmission lines based on substrate integrated waveguides for dual-band and quadband applications", International Journal of Microwave and Wireless Technologies, vol. 5 pp. 213229, June 2013.

[9] S. Lim, C. Caloz, T. Itoh,” Electronically scanned composite right/left handed microstrip leaky-wave antenna”, IEEE Microw. Wirel. Comp. Lett., vol. 14, pp. 277-279, Jun. 2004.

[10] M.A. Antoniades, G.V. Eleftheriades, "A CPS leaky-wave antenna with reduced beam squinting using NRI-TL metamaterials,” IEEE Trans. Antennas Propag., vol. 56, no. 3, pp. 708-721, Mar. 2008.

[11]M. Durán-Sindreu, J. Choi, J. Bonache, F. Martín, T. Itoh "Dual-band leaky wave antenna with filtering capability based on extended-composite right/left-handed transmission lines”, IEEE MTT-S Int. Microwave Symp., June 2013, Seatle (USA). 
[12]G. Zamora, S. Zuffanelli, F. Paredes, F. Javier Herraiz-Martínez, F. Martín, J. Bonache, "Fundamental mode leaky-wave-antenna (LWA) using slot line and split-ring-resonator (SRR) based metamaterials”, IEEE Ant. Wirel. Prop. Lett., vol. 12, pp. 1424-1427, 2013.

[13] J. Bonache, F. Martín, J. García-García, I. Gil, R. Marqués and M. Sorolla, "Ultra wide band pass filtres (UWBPF) based on complementary split rings resonators”, Microwave and Optical Technology Letters, vol. 46, pp.283-286, August 2005.

[14] J. Bonache, I. Gil, J. García-García, F. Martín, "Novel microstrip band pass filters based on complementary split rings resonators", IEEE Transactions on Microwave Theory and Techniques, vol. 54, pp. 265-271, January 2006.

[15] M. Gil, J. Bonache, J. García-García, J. Martel and F. Martín, “Composite right/left handed (crlh) metamaterial transmission lines based on complementary split rings resonators (csrrs) and their applications to very wide band and compact filter design”, IEEE Transactions on Microwave Theory and Techniques, vol. 55, pp. 1296-1304, June 2007.

[16] M. Gil, J. Bonache, F. Martín, “Metamaterial filters: a review”, Metamaterials, vol. 2, pp. 186-197 December 2008.

[17]R. Islam, F. Elek and G.V. Eleftheriades, "Coupled line metamaterial coupler having co-directional phase but contra-directional power flow”, Electronics Letters, vol. 40(5), pp. 315-317, March 2004.

[18] C. Caloz, A. Sanada and T. Itoh, "A novel composite right/left handed coupled-line directional coupler with arbitrary coupling level and broad bandwidth”, IEEE Trans. Microwave Theory Tech., vol. 52, pp. 980-992, March 2004.

[19] M. Durán-Sindreu, A. Vélez, G. Sisó, J. Selga, P. Vélez, J. Bonache, and F. Martín "Recent advances in metamaterial transmission lines based on split rings”, Proceedings of the IEEE, vol. 99, pp. 17011710, October 2011.

[20]F. Martín, F. Falcone, J. Bonache, R. Marqués and M. Sorolla, “Split ring resonator based left handed coplanar waveguide”, Appl. Phys. Lett., vol. 83, pp. 4652-4654, December 2003.

[21]F. Falcone, T. Lopetegi, J.D. Baena, R. Marqués, F. Martín and M. Sorolla, "Effective negative- $\varepsilon$ stop-band microstrip lines based on complementary split ring resonators”, IEEE Microw. Wirel. Comp. Lett., vol. 14, pp. 280-282, Jun. 2004.

[22] M. Durán-Sindreu, A. Vélez, F. Aznar, G. Sisó, J. Bonache and F. Martín, “Application of open split ring resonators and open complementary split ring resonators to the synthesis of artificial transmission lines and microwave passive components”, IEEE Trans. Microwave Theory and Techniques, vol. 57, pp. 3395-3403, Dec. 2009.

[23] C. Caloz and T. Itoh, "Novel microwave devices and structures based on the transmission line approach of metamaterials”, IEEE-MTT Int'l Microwave Symp, vol. 1 Philadelphia, PA, pp. 195-198, June 2003.

[24]F. Aznar, J. Bonache and F. Martín, "Improved circuit model for left handed lines loaded with split ring resonators”, Appl. Phys. Lett., vol. 92, paper 043512, February 2008.

[25] J. Naqui, M. Durán-Sindreu, and F. Martín, “Modeling split ring resonator (SRR) and complementary split ring resonator (CSRR) loaded transmission lines exhibiting cross polarization effects”, IEEE Ant. Wirel. Propag. Lett., vol. 12, pp. 178-181, 2013.

[26] F. Hesmer, E. Tatartschuk, O. Zhuromskyy et al., “Coupling mechanisms for split ring resonators: theory and experiment,” Physica Status Solidi B, vol. 244, pp. 1170-1175, 2007.

[27]E. Tatartschuk, N. Gneiding, F. Hesmer, A. Radkovskaya, and E. Shamonina, "Mapping interelement coupling in metamaterials: scaling down to infrared”, J. Appl. Phys., vol. 111, paper 094904 (2012).

[28]R. R. A. Syms, and L. Solymar, "Effective permeability of a metamaterial: against conventional wisdom”, App. Phys. Lett. vol. 100, paper 124103 (2012).

[29] J. Naqui, A. Fernández-Prieto, F. Mesa, F. Medina and F. Martín, "Effects of inter-resonator coupling in split ring resonator (SRR) loaded metamaterial transmission lines”, J. Appl. Phys., Vol. 115, paper 194903, 2014.

[30] T. Tamir and A. A. Oliner, “Guided complex waves,” Proc. Inst. Elect. Eng., vol. 110, pp. 310-334, 1963.

[31] J. Naqui, M. Durán-Sindreu and F. Martín, "Novel sensors based on the symmetry properties of split ring resonators (SRRs)”, Sensors, vol 11, pp. 7545-7553, 2011.

[32] J. Naqui, C. Damm, A. Wiens, R. Jakoby, L. Su, F. Martín, “Transmission lines loaded with pairs of magnetically coupled stepped impedance resonators: modeling and application to microwave sensors”, IEEE MTT-S Int. Microw. Symp., Tampa, FL (USA), Jun. 2014.

[33] J.B. Pendry, A.J. Holden, D.J. Robbins, W.J. Stewart, "Magnetism from conductors and enhanced nonlinear phenomena”, IEEE Trans. Microwave Theory Tech., vol. 47, pp. 2075-2084, Nov. 1999. 
[34] J. García-García, F. Martín, J.D. Baena, R.Marques, L. Jelinek “On the resonances and polarizabilities of split rings resonators”, J. Applied Physics, vol. 98, pp. 033103-1-9, September 2005.

[35] M. Makimoto and S. Yamashita, “Compact bandpass filters using stepped impedance resonators", Proc. IEEE, Vol. 67 (1), pp. 16-19, Jan 1979.

[36] J-S. Hong and M.J. Lancaster, Microstrip filters for RF/microwave applications, John Wiley, New Jersey, 2001.

[37]F. Aznar, M. Gil, J. Bonache, J.D. Baena, L. Jelinek, R. Marqués, F. Martín, "Characterization of miniaturized metamaterial resonators coupled to planar transmission lines” J. Appl. Phys., vol. 104, paper 114501-1-8, Dec. 2008. 